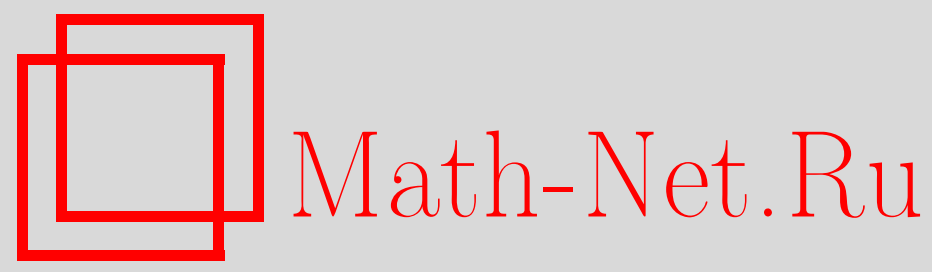

С. В. Смышляев, Булевы функции без предсказывания, Дискрет. матем., 2011, том 23, выпуск 1, 102-118

DOI: https://doi.org/10.4213/dm1134

Использование Общероссийского математического портала Math-Net.Ru подразумевает, что вы прочитали и согласны с пользовательским соглашением http://www.mathnet.ru/rus/agreement

Параметры загрузки:

IP: 52.87 .193 .239

26 апреля 2023 г., $14: 29: 31$ 


\title{
Булевы функции без предсказывания
}

\author{
(c) 2011 г. С. В. Смышляев
}

\begin{abstract}
Рассматриваются свойства булевых функций с барьером конечной длины, предлагается критерий наличия у функции барьера. Вводится понятие булевой функции без предсказывания, описывающее определенные положительные криптографические свойства соответствующих преобразований двоичных последовательностей. Предлагается критерий принадлежности булевой функции классу функций без предсказывания.

Работа выполнена при поддержке Российского фонда фундаментальных исследований, проект 09-01-00653а.
\end{abstract}

\section{1. Введение}

Одним из важных вопросов при изучении класса преобразований двоичных последовательностей, осуществляемых кодирующими устройствами, состоящими из регистра сдвига и булевой функции, является вопрос о наследовании в выходных последовательностях определенных положительных статистических свойств входных последовательностей. Соответствующее свойство используемых в таких кодирующих устройствах булевых функций было определено С. Н. Сумароковым в [2] как сильная равновероятность (мы будем использовать термин совершенная уравновешенность). Также в [2] было предложено важное понятие булевой функции без запрета (функции дефекта нуль), эквивалентное понятию совершенно уравновешенной функции, был получен удобный для теоретических исследований критерий совершенной уравновешенности.

Ряд критериев совершенной уравновешенности, интересных как с точки зрения анализа кодирующих устройств, так и с позиций построения классов совершенно уравновешенных функций, был предложен позже в работах $[4,8,9]$. В работе [4], кроме того, было предложено понятие барьера булевой функции (а также правого и левого барьеров), позволяющее разбить все множество совершенно уравновешенных функций на функции с барьером конечной длины и функции без барьера, была приведена полная классификация совершенно уравновешенных булевых функций от $n \leqslant 5$ переменных, относительно сохраняющих длину барьера преобразований. Свойства функций с барьером позже изучались в работах $[5,7,8,9]$.

В работе [1] была предложена так называемая инверсионная атака на фильтрующие генераторы (криптопримитивы, представляющие собой кодирующие устройства указанного выше типа, на входы которых поступают линейно-рекуррентные последовательности), построенные с помощью линейных по последней существенной переменной функций (функций с барьером длины 1). Идея данной атаки в работе [7] была обобщена на случай функций с барьером произвольной конечной длины, ввиду чего возникла задача нахождения совершенно уравновешенных функций без барьера. Несколько подходов 
к построению широких классов таких функций с использованием предложенной в [3] конструкции было представлено в $[5,6,7]$.

В работе [9], в рамках изучения свойств функций с барьером, были предложены классы частично определенных булевых функций, позволяющих (в определяемом рассматриваемой моделью смысле) восстанавливать входные последовательности кодирующих устройств с имеющими барьер функциями усложнения по выходным последовательностям. В той же работе были доказаны некоторые утверждения о регулярных свойствах множеств двоичных наборов, возникающих при анализе таких частично определенных функций. Часть из этих утверждений косвенно свидетельствует об особых свойствах функций с барьером, изучение которых затруднительно в рамках существующего аппарата работы с классом совершенно уравновешенных функций.

В настоящей работе предлагается и разрабатывается новый аппарат для исследования свойств функций с барьером, предполагающий изучение таких функций с помощью анализа структур множеств двоичных наборов, преобразуемых кодирующими устройствами с имеющими барьер функциями усложнения в одни и те же выходные наборы. С применением данного аппарата устанавливается ряд свойств функций с барьером, предлагается критерий, связывающий наличие у функции барьера со свойствами важного класса преобразований двоичных последовательностей.

Вводится понятие булевой функции без предсказывания, формализующее одно из важных требований к кодирующим устройствам, построенным с помощью регистра сдвига и булевой функции, - невозможность получения информации ни об одном символе выходной последовательности кодирующего устройства по предшествующим выходным символам даже при известном начале входной последовательности, в предположении о независимости и равномерной распределенности символов входной последовательности. В заключительной части работы с использованием полученных результатов о функциях с барьером доказывается утверждение, полностью описывающее класс функций без предсказывания.

\section{2. Определения и предварительные результаты}

Для любого натурального $n$ множество двоичных наборов длины $n$ будем обозначать через $V_{n}=\{0,1\}^{n}$; множество булевых функций от $n$ переменных - через $\mathscr{F}_{n}$.

Пусть $n \geqslant 1, l \geqslant 1, f \in \mathscr{F}_{n}$. Рассмотрим систему булевых уравнений

$$
f\left(x_{s}, x_{s+1}, \ldots, x_{s+n-1}\right)=y_{s}, \quad s=1,2, \ldots, l .
$$

Через $f_{l}$ будем обозначать отображение из $V_{l+n-1}$ в $V_{l}$ следующего вида:

$$
f_{l}\left(x_{1}, x_{2}, \ldots, x_{l+n-1}\right)=\left(f\left(x_{1}, \ldots, x_{n}\right), f\left(x_{2}, \ldots, x_{n+1}\right), \ldots, f\left(x_{l}, \ldots, x_{l+n-1}\right)\right) .
$$

Легко видеть, что отображение $f_{l}$ можно понимать как преобразование, производимое $l$ тактами работы кодирующего устройства, полученного с помощью подключения входов булевой функции $f$ к некоторым ячейкам двоичного регистра сдвига.

Пусть $n \geqslant 1, p \geqslant 1, f \in \mathscr{F}_{n}, l \geqslant \max (1, p-n+2),\left(\tilde{x}_{1}, \tilde{x}_{2}, \ldots, \tilde{x}_{p}\right) \in V_{p}$. Через $f_{l, p}^{\left(\tilde{x}_{1}, \tilde{x}_{2}, \ldots, \tilde{x}_{p}\right)}$ будем обозначать отображение из $V_{l-p+n-1}$ в $V_{l}$, определяемое следующим образом:

$$
f_{l, p}^{\left(\tilde{x}_{1}, \tilde{x}_{2}, \ldots, \tilde{x}_{p}\right)}\left(x_{1}, x_{2}, \ldots, x_{l-p+n-1}\right) \equiv f_{l}\left(\tilde{x}_{1}, \tilde{x}_{2}, \ldots, \tilde{x}_{p}, x_{1}, x_{2}, \ldots, x_{l-p+n-1}\right) .
$$


Отображение $f_{l, p}^{\left(\tilde{x}_{1}, \tilde{x}_{2}, \ldots, \tilde{x}_{p}\right)}$ описывает преобразование, производимое $l$ тактами работы кодирующего устройства с функцией усложнения $f$ на двоичных последовательностях с фиксированным началом из $p$ символов. Особый интерес представляет случай $p=n-1$, при котором отображение данного типа действует из $V_{l}$ в $V_{l}$.

Определение 1 ([2]). Булева функция $f \in \mathscr{F}_{n}$ называется функцией дефекта нуль (функцией без запрета), если соотношение

$$
\left|f_{l}^{-1}(\mathbf{y})\right|>0
$$

выполняется для любого $l \geqslant 1$ и любого $\mathbf{y} \in V_{l}$.

Определение 2 ([2]). Булева функция $f \in \mathscr{F}_{n}$ называется совершенно уравновешенной, если соотношение

$$
\left|f_{l}^{-1}(\mathbf{y})\right|=2^{n-1}
$$

выполняется для любого $l \geqslant 1$ и любого $\mathbf{y} \in V_{l}$. Множество совершенно уравновешенных функций из $\mathscr{F}_{n}$ обозначим через $\mathscr{P}_{\mathscr{P}_{n}}$.

Теорема 1 ([2]). Булева функиия совершенно уравновешена тогда и только тогда, когда она является функцией дефекта нуль (не имеет запрета).

Определение 3. Пусть $\left\{\xi_{i}\right\}_{i=1}^{\infty}-$ бесконечная последовательность независимых в совокупности случайных величин с распределениями

$$
\mathbf{P}\left\{\xi_{i}=0\right\}=\mathbf{P}\left\{\xi_{i}=1\right\}=1 / 2, \quad i=1,2, \ldots
$$

Такую последовательность случайных величин будем называть чисто случайной.

Кодирующие устройства с совершенно уравновешенными функциями усложнения обладают следующим важным свойством: если на вход такого кодирующего устройства поступает чисто случайная последовательность, то выходная последовательность будет также чисто случайной.

Введем понятие барьера булевой функции, тесно связанное с понятием совершенной уравновешенности.

Определение 4 ([4]). Булева функция $f \in \mathscr{F}_{n}$ называется функцией с правым барьером длины $b$, если система уравнений

$$
\begin{aligned}
f\left(x_{1}, x_{2}, \ldots, x_{n}\right) & =f\left(z_{1}, z_{2}, \ldots, z_{n}\right) \\
f\left(x_{2}, x_{3}, \ldots, x_{n+1}\right) & =f\left(z_{2}, z_{3}, \ldots, z_{n+1}\right) \\
& \ldots \\
f\left(x_{b-1}, x_{b}, \ldots, x_{b+n-2}\right) & =f\left(z_{b-1}, z_{b}, \ldots, z_{b+n-2}\right) \\
x_{1}=z_{1}, \ldots, x_{n-1} & =z_{n-1}, x_{n}=0, z_{n}=1
\end{aligned}
$$

имеет решение, а система

$$
\begin{aligned}
f\left(x_{1}, x_{2}, \ldots, x_{n}\right) & =f\left(z_{1}, z_{2}, \ldots, z_{n}\right) \\
f\left(x_{2}, x_{3}, \ldots, x_{n+1}\right) & =f\left(z_{2}, z_{3}, \ldots, z_{n+1}\right) \\
& \ldots \\
f\left(x_{b-1}, x_{b}, \ldots, x_{b+n-2}\right) & =f\left(z_{b-1}, z_{b}, \ldots, z_{b+n-2}\right) \\
f\left(x_{b}, x_{b+1}, \ldots, x_{b+n-1}\right) & =f\left(z_{b}, z_{b+1}, \ldots, z_{b+n-1}\right) \\
x_{1}=z_{1}, \ldots, x_{n-1} & =z_{n-1}, x_{n}=0, z_{n}=1
\end{aligned}
$$


решений не имеет.

Булева функция $f \in \mathscr{F}_{n}$ называется функцией с левым барьером длины $b$, если $f^{\prime}\left(x_{1}, \ldots, x_{n}\right) \equiv f\left(x_{n}, \ldots, x_{1}\right)$ является функцией с правым барьером длины $b$.

Булева функция $f \in \mathscr{F}_{n}$ имеет барьер, если она имеет правый или левый барьер, или оба сразу. При этом длиной барьера функции называется соответственно длина правого барьера, левого барьера, или меньшая из длин барьеров.

Связь совершенной уравновешенности с наличием у функции барьера описывается следующим утверждением.

Теорема 2 ([4]). Если функиия имеет барьер, то она совершенно уравновешена.

Замечание 1. В работе [4] показано, что наличие барьера не является необходимым условием совершенной уравновешенности; в работе [6] приведен метод построения широких классов совершенно уравновешенных булевых функций без барьера.

Отметим, что для всех утверждений, относящихся к функциям с правым барьером, очевидным образом могут быть построены аналоги для функций с левым барьером. Ввиду этого, далее будем говорить только о правых барьерах функций, обозначая для удобства длину правого барьера булевой функции $f$ через $b_{f}^{R}$.

Замечание 2. Нетрудно заметить, что наличие правого барьера длины $b_{f}^{R}=1$ означает линейность функции по последнему аргументу.

\section{3. Основные результаты}

Как видно из результатов работ $[5,7,8]$, булевы функции с правым барьером обладают рядом особых свойств, определенным образом характеризующих свойства выходных последовательностей кодирующих устройств с такими функциями усложнения. Рассмотрим вопрос о свойствах отображений двоичных последовательностей, осуществляемых кодирующими устройствами с имеющими правый барьер функциями усложнения, в случае фиксированного начального заполнения регистра.

Нам понадобится следующее вспомогательное утверждение.

Лемма 1. Пусть $f \in \mathscr{F}_{n}$. Для любого $r \geqslant 1$ илюбых наборов $\left(\tilde{x}_{1}, \tilde{x}_{2}, \ldots, \tilde{x}_{n-1}\right) \in V_{n-1}$, $\left(y_{1}, y_{2}, \ldots, y_{r}\right) \in V_{r}$ справедливо соотношение

$$
\begin{aligned}
\begin{aligned}
\left|f_{r+1, n-1}^{\left(\tilde{x}_{1}, \tilde{x}_{2}, \ldots, \tilde{x}_{n-1}\right)^{-1}}\left(y_{1}, y_{2}, \ldots, y_{r}, 0\right)\right|+\left|f_{r+1, n-1}^{\left(\tilde{x}_{1}, \tilde{x}_{2}, \ldots, \tilde{x}_{n-1}\right)^{-1}}\left(y_{1}, y_{2}, \ldots, y_{r}, 1\right)\right| \\
=2\left|f_{r, n-1}^{\left(\tilde{x}_{1}, \tilde{x}_{2}, \ldots, \tilde{x}_{n-1}\right)^{-1}}\left(y_{1}, y_{2}, \ldots, y_{r}\right)\right| .
\end{aligned}
\end{aligned}
$$

Доказательство. Требуемое равенство следует из очевидных соотношений

$$
f_{r+1, n-1}^{\left(\tilde{x}_{1}, \tilde{x}_{2}, \ldots, \tilde{x}_{n-1}\right)^{-1}}\left(y_{1}, y_{2}, \ldots, y_{r}, 0\right) \cap f_{r+1, n-1}^{\left(\tilde{x}_{1}, \tilde{x}_{2}, \ldots, \tilde{x}_{n-1}\right)^{-1}}\left(y_{1}, y_{2}, \ldots, y_{r}, 1\right)=\varnothing
$$

и

$$
\begin{aligned}
& f_{r+1, n-1}^{\left(\tilde{x}_{1}, \tilde{x}_{2}, \ldots, \tilde{x}_{n-1}\right)^{-1}}\left(y_{1}, y_{2}, \ldots, y_{r}, 0\right) \cup f_{r+1, n-1}^{\left(\tilde{x}_{1}, \tilde{x}_{2}, \ldots, \tilde{x}_{n-1}\right)^{-1}}\left(y_{1}, y_{2}, \ldots, y_{r}, 1\right) \\
& \quad=\left\{\left(x_{1}, \ldots, x_{r}, t\right) \in V_{r+1} \mid f_{r, n-1}^{\left(\tilde{x}_{1}, \ldots, \tilde{x}_{n-1}\right)}\left(x_{1}, \ldots, x_{r}\right)=\left(y_{1}, \ldots, y_{r}\right), t \in\{0,1\}\right\} .
\end{aligned}
$$


Теорема 3. Для любой функции $f \in \mathscr{F}_{n}$ с правым барьером существует такое число $c, c=c(f)$, что для любого $l \geqslant b_{f}^{R}-1$ илюбых наборов $\left(x_{1}, x_{2}, \ldots, x_{n-1}\right) \in V_{n-1}$, $\left(y_{1}, y_{2}, \ldots, y_{l}\right) \in V_{l}$ выполняется одно из двух равенств:

$$
\left|f_{l, n-1}^{\left(x_{1}, x_{2}, \ldots, x_{n-1}\right)^{-1}}\left(y_{1}, y_{2}, \ldots, y_{l}\right)\right|=0
$$

или

$$
\left|f_{l, n-1}^{\left(x_{1}, x_{2}, \ldots, x_{n-1}\right)^{-1}}\left(y_{1}, y_{2}, \ldots, y_{l}\right)\right|=c .
$$

Доказательство. Выберем произвольные $l^{\prime} \geqslant b_{f}^{R}-1, l^{\prime \prime} \geqslant b_{f}^{R}-1$. Пусть

$$
\begin{aligned}
\left(x_{1}^{\prime}, x_{2}^{\prime}, \ldots, x_{n-1}^{\prime}\right) & \in V_{n-1}, & \left(x_{1}^{\prime \prime}, x_{2}^{\prime \prime}, \ldots, x_{n-1}^{\prime \prime}\right) & \in V_{n-1}, \\
\left(y_{1}^{\prime}, y_{2}^{\prime}, \ldots, y_{l^{\prime}}^{\prime}\right) & \in V_{l^{\prime}}, & \left(y_{1}^{\prime \prime}, y_{2}^{\prime \prime}, \ldots, y_{l^{\prime \prime}}^{\prime \prime}\right) & \in V_{l^{\prime \prime}}
\end{aligned}
$$

таковы, что

$$
\begin{aligned}
& \left|f_{l^{\prime}, n-1}^{\left(x_{1}^{\prime}, x_{2}^{\prime}, \ldots, x_{n-1}^{\prime}\right)^{-1}}\left(y_{1}^{\prime}, y_{2}^{\prime}, \ldots, y_{l^{\prime}}^{\prime}\right)\right|>0, \\
& \left|f_{l^{\prime \prime}, n-1}^{\left(x_{1}^{\prime \prime}, x_{2}^{\prime \prime}, \ldots, x_{n-1}^{\prime \prime}\right)^{-1}}\left(y_{1}^{\prime \prime}, y_{2}^{\prime \prime}, \ldots, y_{l^{\prime \prime}}^{\prime \prime}\right)\right|>0 .
\end{aligned}
$$

Докажем, что выполняется равенство

$$
\left|f_{l^{\prime}, n-1}^{\left(x_{1}^{\prime}, x_{2}^{\prime}, \ldots, x_{n-1}^{\prime}\right)^{-1}}\left(y_{1}^{\prime}, y_{2}^{\prime}, \ldots, y_{l^{\prime}}^{\prime}\right)\right|=\left|f_{l^{\prime \prime}, n-1}^{\left(x_{1}^{\prime \prime}, x_{2}^{\prime \prime}, \ldots, x_{n-1}^{\prime \prime}\right)^{-1}}\left(y_{1}^{\prime \prime}, y_{2}^{\prime \prime}, \ldots, y_{l^{\prime \prime}}^{\prime \prime}\right)\right| .
$$

Зафиксируем произвольным образом набор $\left(\tilde{x}_{1}, \tilde{x}_{2}, \ldots, \tilde{x}_{n-1}\right)$ и рассмотрим величину

$$
M=\max _{r \geqslant 1,\left(y_{1}, y_{2}, \ldots, y_{r}\right) \in V_{r}}\left|f_{r, n-1}^{\left(\tilde{x}_{1}, \tilde{x}_{2}, \ldots, \tilde{x}_{n-1}\right)^{-1}}\left(y_{1}, y_{2}, \ldots, y_{r}\right)\right| .
$$

Непосредственно из определения правого барьера длины $b_{f}^{R}$ следует (см. [7]), что любые два набора из множества $f_{r, n-1}^{\left(\tilde{x}_{1}, \tilde{x}_{2}, \ldots, \tilde{x}_{n-1}\right)^{-1}}\left(y_{1}, y_{2}, \ldots, y_{r}\right)$ при произвольных $r \geqslant 1$ и $\left(y_{1}, y_{2}, \ldots, y_{r}\right) \in V_{r}$ могут различаться между собой только в последних $b_{f}^{R}-1$ символах. Следовательно, величина $M$ определена и не превосходит $2^{b_{f}^{R}-1}$.

Пусть максимум в (6) достигается при $r=r^{*}$ и $\left(y_{1}, y_{2}, \ldots, y_{r}\right)=\left(y_{1}^{*}, y_{2}^{*}, \ldots, y_{r^{*}}^{*}\right)$, то есть, для любого $r \geqslant 1$ и любого набора $\left(y_{1}, y_{2}, \ldots, y_{r}\right) \in V_{r}$ верно соотношение

$$
\left|f_{r, n-1}^{\left(\tilde{x}_{1}, \tilde{x}_{2}, \ldots, \tilde{x}_{n-1}\right)^{-1}}\left(y_{1}, y_{2}, \ldots, y_{r}\right)\right| \leqslant\left|f_{r^{*}, n-1}^{\left(\tilde{x}_{1}, \tilde{x}_{2}, \ldots, \tilde{x}_{n-1}\right)^{-1}}\left(y_{1}^{*}, y_{2}^{*}, \ldots, y_{r^{*}}^{*}\right)\right| .
$$

Используя это соотношение, а также лемму 1 , индукцией по $r, r=r^{*}, r^{*}+1, \ldots$, легко показать, что в таком случае равенство

$$
\left|f_{r, n-1}^{\left(\tilde{x}_{1}, \tilde{x}_{2}, \ldots, \tilde{x}_{n-1}\right)^{-1}}\left(y_{1}^{*}, y_{2}^{*}, \ldots, y_{r^{*}}^{*}, y_{r^{*}+1}, y_{r^{*}+2}, \ldots, y_{r}\right)\right|=M
$$

выполняется при любых $r \geqslant r^{*}$ и $\left(y_{r^{*}+1}, y_{r^{*}+2}, \ldots, y_{r}\right) \in V_{r-r^{*}}$.

Очевидно, $M \geqslant 1$; зафиксируем любой набор $\left(\tilde{x}_{n}, \tilde{x}_{n+1}, \ldots, \tilde{x}_{r^{*}+n-1}\right)$ из множества $f_{r^{*}, n-1}^{\left(\tilde{x}_{1}, \tilde{x}_{2}, \ldots, \tilde{x}_{n-1}\right)^{-1}}\left(y_{1}^{*}, y_{2}^{*}, \ldots, y_{r^{*}}^{*}\right)$. Пусть

$$
\begin{aligned}
\left(y_{r^{*}+1}^{\prime *}, y_{r^{*}+2}^{\prime *}, \ldots, y_{r^{*}+n-1}^{\prime *}\right) & =f_{n-1}\left(\tilde{x}_{r^{*}+1}, \tilde{x}_{r^{*}+2}, \ldots, \tilde{x}_{r^{*}+n-1}, x_{1}^{\prime}, x_{2}^{\prime}, \ldots, x_{n-1}^{\prime}\right), \\
\left(y_{r^{*}+1}^{\prime \prime *}, y_{r^{*}+2}^{\prime \prime *}, \ldots, y_{r^{*}+n-1}^{\prime \prime *}\right) & =f_{n-1}\left(\tilde{x}_{r^{*}+1}, \tilde{x}_{r^{*}+2}, \ldots, \tilde{x}_{r^{*}+n-1}, x_{1}^{\prime \prime}, x_{2}^{\prime \prime}, \ldots, x_{n-1}^{\prime \prime}\right) .
\end{aligned}
$$

Далее для доказательства нам потребуется следующее утверждение. 
Лемма 2. Для определенных вышеописанным образом наборов выполняются соотношения

$$
\begin{aligned}
& \left|f_{l^{\prime}, n-1}^{\left(x_{1}^{\prime}, x_{2}^{\prime}, \ldots, x_{n-1}^{\prime}\right)^{-1}}\left(y_{1}^{\prime}, y_{2}^{\prime}, \ldots, y_{l^{\prime}}^{\prime}\right)\right| \\
& =\left|f_{r^{*}+n-1+l^{\prime}, n-1}^{\left(\tilde{x}_{1}, \tilde{x}_{2}, \ldots, \tilde{x}_{n-1}\right)^{-1}}\left(y_{1}^{*}, \ldots, y_{r^{*}}^{*}, y_{r^{*}+1}^{\prime *}, y_{r^{*}+2}^{\prime *}, \ldots, y_{r^{*}+n-1}^{\prime *}, y_{1}^{\prime}, y_{2}^{\prime}, \ldots, y_{l^{\prime}}^{\prime}\right)\right|
\end{aligned}
$$

$u$

$$
\begin{aligned}
& \left|f_{l^{\prime \prime}, n-1}^{\left(x_{1}^{\prime \prime}, x_{2}^{\prime \prime}, \ldots, x_{n-1}^{\prime \prime}\right)^{-1}}\left(y_{1}^{\prime \prime}, y_{2}^{\prime \prime}, \ldots, y_{l^{\prime \prime}}^{\prime \prime}\right)\right| \\
& =\left|f_{r^{*}+n-1+l^{\prime \prime}, n-1}^{\left(\tilde{x}_{1}, \tilde{x}_{2}, \ldots, \tilde{x}_{n-1}\right)}{ }^{-1}\left(y_{1}^{*}, \ldots, y_{r^{*}}^{*}, y_{r^{*}+1}^{\prime \prime}, y_{r^{*}+2}^{\prime \prime *}, \ldots, y_{r^{*}+n-1}^{\prime *}, y_{1}^{\prime \prime}, y_{2}^{\prime \prime}, \ldots, y_{l^{\prime \prime}}^{\prime \prime}\right)\right| .
\end{aligned}
$$

Доказательство. Зафиксируем некоторые наборы

$$
\begin{aligned}
&\left(x_{n}^{\prime}, x_{n+1}^{\prime}, \ldots, x_{l^{\prime}+n-1}^{\prime}\right) \in f_{l^{\prime}, n-1}^{\left(x_{1}^{\prime}, x_{2}^{\prime}, \ldots, x_{n-1}^{\prime}\right)^{-1}}\left(y_{1}^{\prime}, y_{2}^{\prime}, \ldots, y_{l^{\prime}}^{\prime}\right), \\
&\left(x_{n}^{\prime \prime}, x_{n+1}^{\prime \prime}, \ldots, x_{l^{\prime \prime}+n-1}^{\prime \prime}\right) \in f_{l^{\prime \prime}, n-1}^{\left(x_{1}^{\prime \prime}, x_{2}^{\prime \prime}, \ldots, x_{n-1}^{\prime \prime}\right)^{-1}}\left(y_{1}^{\prime \prime}, y_{2}^{\prime \prime}, \ldots, y_{l^{\prime \prime}}^{\prime \prime}\right) .
\end{aligned}
$$

Из определения правого барьера длины $b_{f}^{R}$ следует, что входящие в множество $f_{r^{*}+n-1+l^{\prime}, n-1}^{\left(\tilde{x}_{1}, \tilde{x}_{2}, \ldots, \tilde{x}_{n-1}\right)^{-1}}\left(y_{1}^{*}, y_{2}^{*}, \ldots, y_{r^{*}}^{*}, y_{r^{*}+1}^{\prime *}, y_{r^{*}+2}^{\prime *}, \ldots, y_{r^{*}+n-1}^{\prime *}, y_{1}^{\prime}, y_{2}^{\prime}, \ldots, y_{l^{\prime}}^{\prime}\right) \quad$ наборы $\left(x_{n}, x_{n+1}, \ldots, x_{\left.r^{*}+l^{\prime}+2 n-2\right)}\right.$ различаются только в последних $b_{f}^{R}-1$ символах. Заметим, что указанное множество непусто, так как в него, по построению, входит, набор $\left(\tilde{x}_{n}, \tilde{x}_{n+1}, \ldots, \tilde{x}_{r^{*}+n-1}, x_{1}^{\prime}, x_{2}^{\prime}, \ldots, x_{n-1}^{\prime}, x_{n}^{\prime}, \ldots, x_{l^{\prime}+n-1}^{\prime}\right)$. Выполняется соотношение $l^{\prime} \geqslant b_{f}^{R}-1$, поэтому

$$
\begin{gathered}
f_{r^{*}+n-1+l^{\prime}, n-1}^{\left(\tilde{x}_{1}, \tilde{x}_{2}, \ldots, \tilde{x}_{n-1}\right)^{-1}}\left(y_{1}^{*}, y_{2}^{*}, \ldots, y_{r^{*}}^{*}, y_{r^{*}+1}^{\prime *}, y_{r^{*}+2}^{\prime *}, \ldots, y_{r^{*}+n-1}^{\prime *}, y_{1}^{\prime}, y_{2}^{\prime}, \ldots, y_{l^{\prime}}^{\prime}\right) \\
=\left\{\left(x_{n}, x_{n+1}, \ldots, x_{r^{*}+l^{\prime}+2 n-2}\right) \in V_{r^{*}+l^{\prime}+n-1} \mid x_{n}=\tilde{x}_{n}, x_{n+1}=\tilde{x}_{n+1}, \ldots,\right. \\
x_{r^{*}+n-1}=\tilde{x}_{r^{*}+n-1}, x_{r^{*}+n}=x_{1}^{\prime}, x_{r^{*}+n+1}=x_{2}^{\prime}, \ldots, x_{r^{*}+2 n-2}=x_{n-1}^{\prime}, \\
\left.f_{l^{\prime}, n-1}^{\left(x_{1}^{\prime}, x_{2}^{\prime}, \ldots, x_{n-1}^{\prime}\right)}\left(x_{r^{*}+2 n-1}, \ldots, x_{r^{*}+l^{\prime}+2 n-2}\right)=\left(y_{1}^{\prime}, y_{2}^{\prime}, \ldots, y_{l^{\prime}}^{\prime}\right)\right\} .
\end{gathered}
$$

Рассуждая аналогично, получим, что

$$
\begin{gathered}
f_{r^{*}+n-1+l^{\prime \prime}, n-1}^{\left(\tilde{x}_{1}, \tilde{x}_{2}, \ldots, \tilde{x}_{n-1}\right)^{-1}}\left(y_{1}^{*}, y_{2}^{*}, \ldots, y_{r^{*}}^{*}, y_{r^{*}+1}^{\prime \prime *}, y_{r^{*}+2}^{\prime \prime *}, \ldots, y_{r^{*}+n-1}^{\prime \prime *}, y_{1}^{\prime \prime}, y_{2}^{\prime \prime}, \ldots, y_{l^{\prime \prime}}^{\prime \prime}\right) \\
=\left\{\left(x_{n}, x_{n+1}, \ldots, x_{r^{*}+l^{\prime \prime}+2 n-2}\right) \in V_{r^{*}+l^{\prime \prime}+n-1} \mid x_{n}=\tilde{x}_{n}, x_{n+1}=\tilde{x}_{n+1}, \ldots,\right. \\
\quad x_{r^{*}+n-1}=\tilde{x}_{r^{*}+n-1}, x_{r^{*}+n}=x_{1}^{\prime \prime}, x_{r^{*}+n+1}=x_{2}^{\prime \prime}, \ldots, x_{r^{*}+2 n-2}=x_{n-1}^{\prime \prime}, \\
\left.f_{l^{\prime \prime}, n-1}^{\left(x_{1}^{\prime \prime}, x_{2}^{\prime \prime}, \ldots, x_{n-1}^{\prime \prime}\right)}\left(x_{r^{*}+2 n-1}, \ldots, x_{r^{*}+l^{\prime \prime}+2 n-2}\right)=\left(y_{1}^{\prime \prime}, y_{2}^{\prime \prime}, \ldots, y_{l^{\prime \prime}}^{\prime \prime}\right)\right\} .
\end{gathered}
$$

Из (10) и (11) следуют требуемые соотношения (8) и (9).

Учитывая (7) и доказанные в лемме 2 равенства (8) и (9), получим, что

$$
\begin{aligned}
& \left|f_{l^{\prime}, n-1}^{\left(x_{1}^{\prime}, x_{2}^{\prime}, \ldots, x_{n-1}^{\prime}\right)^{-1}}\left(y_{1}^{\prime}, y_{2}^{\prime}, \ldots, y_{l^{\prime}}^{\prime}\right)\right| \\
& =\mid f_{r^{*}+n-1+l^{\prime}, n-1}^{\left(\tilde{x}_{1}, \tilde{x}_{2}, \ldots, \tilde{x}_{n-1}\right)}-1 \\
& \left.=\mid y_{1}^{*}, y_{2}^{*}, \ldots, y_{r^{*}}^{*}, y_{r^{*}+1}^{*}, y_{r^{*}+2}^{*}, \ldots, y_{r^{*}+n-1}^{*}, y_{1}^{\prime}, y_{2}^{\prime}, \ldots, y_{l^{\prime}}^{\prime}\right) \mid=M \\
& \left.=\mid f_{\left.r^{*}+n-1+\tilde{x}_{2}, \ldots, \tilde{x}_{n-1}\right)}^{\prime}{ }^{-1}\left(y_{1}^{\prime \prime}, x_{2}^{\prime \prime}, \ldots, x_{n-1}^{\prime \prime}\right)^{-1}, y_{2}^{*}, \ldots, y_{r^{*}}^{*}, y_{r^{*}+1}^{\prime \prime}, y_{r^{*}+2}^{*}, \ldots, y_{r^{*}+n-1}^{\prime \prime}, y_{1}^{\prime \prime}, y_{2}^{\prime \prime}, \ldots, y_{l^{\prime \prime}}^{\prime \prime}\right) \mid
\end{aligned}
$$


Другими словами, если при каких-то $l^{\prime} \geqslant b_{f}^{R}-1, l^{\prime \prime} \geqslant b_{f}^{R}-1,\left(x_{1}^{\prime}, x_{2}^{\prime}, \ldots, x_{n-1}^{\prime}\right) \in V_{n-1}$, $\left(x_{1}^{\prime \prime}, x_{2}^{\prime \prime}, \ldots, x_{n-1}^{\prime \prime}\right) \in V_{n-1},\left(y_{1}^{\prime}, y_{2}^{\prime}, \ldots, y_{l^{\prime}}^{\prime}\right) \in V_{l^{\prime}},\left(y_{1}^{\prime \prime}, y_{2}^{\prime \prime}, \ldots, y_{l^{\prime \prime}}^{\prime \prime}\right) \in V_{l^{\prime \prime}}$, для величин

$$
\begin{aligned}
& c_{1}=\left|f_{l^{\prime}, n-1}^{\left(x_{1}^{\prime}, x_{2}^{\prime}, \ldots, x_{n-1}^{\prime}\right)^{-1}}\left(y_{1}^{\prime}, y_{2}^{\prime}, \ldots, y_{l^{\prime}}^{\prime}\right)\right|, \\
& c_{2}=\left|f_{l^{\prime \prime}, n-1}^{\left(x_{1}^{\prime \prime}, x_{2}^{\prime \prime}, \ldots, x_{n-1}^{\prime \prime}\right)^{-1}}\left(y_{1}^{\prime \prime}, y_{2}^{\prime \prime}, \ldots, y_{l^{\prime \prime}}^{\prime \prime}\right)\right|
\end{aligned}
$$

выполняются соотношения $c_{1} \neq 0, c_{2} \neq 0$, то $c_{1}=c_{2}$. Таким образом, существует постоянная $c$, зависящая только от самой функции $f$, такая, что для любых $l \geqslant b_{f}^{R}-1$, $\left(x_{1}, x_{2}, \ldots, x_{n-1}\right) \in V_{n-1},\left(y_{1}, y_{2}, \ldots, y_{l}\right) \in V_{l}$ выполняется либо равенство (4), либо равенство (5).

Теорема 4. Для каждой функции $f \in \mathscr{F}_{n}$ с правым барьером можно определить величину $e_{f}^{R} \in\left\{0,1, \ldots, b_{f}^{R}-1\right\}$ такую, что для любых $p \geqslant n-1, l \geqslant b_{f}^{R}+p-n,\left(\tilde{x}_{1}, \tilde{x}_{2}, \ldots, \tilde{x}_{p}\right) \in V_{p}$ и любого набора $\left(y_{1}, y_{2}, \ldots, y_{l}\right) \in \operatorname{Im}\left(f_{l, p}^{\left(\tilde{x}_{1}, \tilde{x}_{2}, \ldots, \tilde{x}_{p}\right)}\right)$ выполняется равенство

$$
\left|f_{l, p}^{\left(\tilde{x}_{1}, \tilde{x}_{2}, \ldots, \tilde{x}_{p}\right)^{-1}}\left(y_{1}, y_{2}, \ldots, y_{l}\right)\right|=2^{e_{f}^{R}} .
$$

Доказательство. Проведем доказательство индукцией по $p, p=n-1, n, n+1, \ldots$

Выберем базис индукции. Пусть $p=n-1$. Как следует из теоремы 3 , для произвольной функции $f \in \mathscr{F}_{n}$ с правым барьером длины $b_{f}^{R}$ существует $c \geqslant 1, c=c(f)$, такое, что для любых $l \geqslant b_{f}^{R}+(n-1)-n,\left(\tilde{x}_{1}, \tilde{x}_{2}, \ldots, \tilde{x}_{n-1}\right) \in V_{n-1}$ и любого $\left(y_{1}, y_{2}, \ldots, y_{l}\right) \in$ $\operatorname{Im}\left(f_{l, n-1}^{\left(\tilde{x}_{1}, \tilde{x}_{2}, \ldots, \tilde{x}_{n-1}\right)}\right)$ выполняется равенство

$$
\left|f_{l, n-1}^{\left(\tilde{x}_{1}, \tilde{x}_{2}, \ldots, \tilde{x}_{n-1}\right)^{-1}}\left(y_{1}, y_{2}, \ldots, y_{l}\right)\right|=c .
$$

Требуется показать, что

$$
c=2^{e_{f}^{R}}, \quad e_{f}^{R} \in\left\{0,1,2, \ldots, b_{f}^{R}-1\right\} .
$$

Для любых различных наборов $\mathbf{y}^{\prime} \in V_{l}, \mathbf{y}^{\prime \prime} \in V_{l}$ верно, что множества $f_{l, n-1}^{\left(\tilde{x}_{1}, \tilde{x}_{2}, \ldots, \tilde{x}_{n-1}\right)^{-1}}\left(\mathbf{y}^{\prime}\right)$ и $f_{l, n-1}^{\left(\tilde{x}_{1}, \tilde{x}_{2}, \ldots, \tilde{x}_{n-1}\right)^{-1}}\left(\mathbf{y}^{\prime \prime}\right)$ не пересекаются, при этом, очевидно, что

$$
\bigcup_{\mathbf{y} \in V_{l}} f_{l, n-1}^{\left(\tilde{x}_{1}, \tilde{x}_{2}, \ldots, \tilde{x}_{n-1}\right)^{-1}}(\mathbf{y})=V_{l},
$$

откуда

$$
\sum_{\mathbf{y} \in V_{l}}\left|f_{l, n-1}^{\left(\tilde{x}_{1}, \tilde{x}_{2}, \ldots, \tilde{x}_{n-1}\right)^{-1}}(\mathbf{y})\right|=2^{l} .
$$

Как следует из последнего равенства и (13),

$$
c\left|\operatorname{Im}\left(f_{l, n-1}^{\left(\tilde{x}_{1}, \tilde{x}_{2}, \ldots, \tilde{x}_{n-1}\right)}\right)\right|=2^{l},
$$

поэтому $c=2^{e_{f}^{R}}$ для некоторого $e_{f}^{R} \in\{0,1, \ldots, l\}$.

Учитывая, что равенство (14) выполняется для произвольного $l \geqslant b_{f}^{R}-1$, получим, что

$$
e_{f}^{R} \leqslant b_{f}^{R}-1
$$


Проведем индуктивный переход. Пусть условие теоремы выполнено для $p=p^{*}$, покажем, что в таком случае оно выполнено и для $p=p^{*}+1, p^{*}+1 \geqslant n$. Зафиксируем $l$,

$$
l \geqslant b_{f}^{R}+p-n=b_{f}^{R}+p^{*}+1-n,
$$

где $\left(\tilde{x}_{1}, \tilde{x}_{2}, \ldots, \tilde{x}_{p^{*}+1}\right) \in V_{p^{*}+1} \quad$ и $\left(y_{1}, y_{2}, \ldots, y_{l}\right) \in \operatorname{Im}\left(f_{l, p^{*}+1}^{\left(\tilde{x}_{1}, \tilde{x}_{2}, \ldots, \tilde{x}_{p^{*}+1}\right)}\right)$. Так как $\left(y_{1}, y_{2}, \ldots, y_{l}\right) \in \operatorname{Im}\left(f_{l, p^{*}+1}^{\left(\tilde{x}_{1}, \tilde{x}_{2}, \ldots, \tilde{x}_{p^{*}+1}\right)}\right)$ и $p^{*}+1 \geqslant n$, то $y_{1}=f\left(\tilde{x}_{1}, \tilde{x}_{2}, \ldots, \tilde{x}_{n}\right)$. Поэтому мощность множества $f_{l, p^{*}+1}^{\left(\tilde{x}_{1}, \tilde{x}_{2}, \ldots, \tilde{x}_{p^{*}+1}\right)^{-1}}\left(y_{1}, y_{2}, \ldots, y_{l}\right)$ равна мощности множества $f_{l-1, p^{*}}^{\left(\tilde{x}_{2}, \tilde{x}_{3}, \ldots, \tilde{x}_{p^{*}+1}\right)^{-1}}\left(y_{2}, \ldots, y_{l}\right)$, которая, в свою очередь, по предположению индукции, так как

$$
l-1 \geqslant b_{f}^{R}+p^{*}+1-n-1=b_{f}^{R}+p^{*}-n,
$$

равна $2_{f}^{e_{f}^{R}}$.

Замечание 3. С учетом того, что любая функция с правым барьером является совершенно уравновешенной, легко показать, что для всех функций с правым барьером выполняется неравенство $e_{f}^{R} \leqslant n-1$, даже в случае $b_{f}^{R}>n$.

Возвращаясь к равенству (14), легко получить следующее важное следствие.

Следствие 1. Пусть $f \in \mathscr{F}_{n}$ имеет правый барьер. Для любых $l \geqslant b_{f}^{R}-1$ и любого набора $\left(\tilde{x}_{1}, \tilde{x}_{2}, \ldots, \tilde{x}_{n-1}\right) \in V_{n-1}$ справедливо равенство

$$
\left|\operatorname{Im}\left(f_{l, n-1}^{\left(\tilde{x}_{1}, \tilde{x}_{2}, \ldots, \tilde{x}_{n-1}\right)}\right)\right|=2^{l-e_{f}^{R}} .
$$

Для любой $f \in \mathscr{F}_{n}$ и любых $l \geqslant 1,\left(y_{1}, y_{2}, \ldots, y_{l}\right) \in V_{l}$ введем следующее обозначение:

$$
A_{\left(y_{1}, y_{2}, \ldots, y_{l}\right)}^{f}=\left\{\left(x_{1}, x_{2}, \ldots, x_{n-1}\right) \in V_{n-1} \mid f_{l, n-1}^{\left(x_{1}, x_{2}, \ldots, x_{n-1}\right)^{-1}}\left(y_{1}, y_{2}, \ldots, y_{l}\right) \neq \varnothing\right\}
$$

Следствие 2. Пусть $f \in \mathscr{F}_{n}$ имеет правый барьер. Для любого $l \geqslant b_{f}^{R}-1$ и любого набора $\left(y_{1}, y_{2}, \ldots, y_{l}\right) \in V_{l}$ верно равенство

$$
\left|A_{\left(y_{1}, y_{2}, \ldots, y_{l}\right)}^{f}\right|=2^{n-1-e_{f}^{R}} .
$$

Доказательство. Фиксируем $l, l \geqslant b_{f}^{R}-1$, и набор $\mathbf{y}=\left(y_{1}, y_{2}, \ldots, y_{l}\right) \in V_{l}$. Функция $f \in \mathscr{F}_{n}$ имеет правый барьер, следовательно, $f \in \mathscr{P}_{\mathscr{B}_{n}}$ и $\left|f_{l}^{-1}(\mathbf{y})\right|=2^{n-1}$. Согласно теореме 4 , для любого $\left(x_{1}, x_{2}, \ldots, x_{n-1}\right) \in A_{\mathbf{y}}^{f}$ справедливо равенство

$$
\left|f_{l, n-1}^{\left(x_{1}, x_{2}, \ldots, x_{n-1}\right)^{-1}}(\mathbf{y})\right|=2^{e_{f}^{R}} .
$$

Таким образом, справедливо равенство

$$
2^{n-1}=\sum_{\mathbf{x} \in A_{\mathbf{y}}^{f}} 2^{e_{f}^{R}}=\left|A_{\mathbf{y}}^{f}\right| \cdot 2_{f}^{e_{f}^{R}},
$$

из которого и следует (16). 
Следствие 3. Пусть $f\left(x_{1}, x_{2}, \ldots, x_{n}\right) \in \mathscr{F}_{n}$ имеет правый барьер. Тогда равенство

$$
e_{f}^{R}=b_{f}^{R}-1
$$

справедливо тогда и только тогда, когда функция $f$ не зависит сущчественно от переменных $x_{n-b_{f}^{R}+2}, x_{n-b_{f}^{R}+3}, \ldots, x_{n}$ и линейна по переменной $x_{n-b_{f}^{R}+1}$.

Доказательство. Из результатов [7] следует, что при $l \geqslant b_{f}^{R}-1$ набор $\left(y_{1}, y_{2}, \ldots, y_{l}\right)$ такой, что выполняется равенство

$$
\left|A_{\left(y_{1}, y_{2}, \ldots, y_{l}\right)}^{f}\right|=2^{n-b_{f}^{R}}
$$

существует в том и только в том случае, когда $f$ не зависит существенно от переменных $x_{n-b_{f}^{R}+2}, x_{n-b_{f}^{R}+3}, \ldots, x_{n}$ и линейна по переменной $x_{n-b_{f}^{R}+1}$. Отсюда, с учетом (16), получаем требуемое утверждение.

Легко можно описать и множество функций с $e_{f}^{R}=0$.

Предложение 1. Если функичия $f \in \mathscr{F}_{n}$ имеет правый барьер, то $e_{f}^{R}=0$ тогда и только тогда, когда $b_{f}^{R}=1$.

Доказательство. Если $b_{f}^{R}=1$, то равенство $e_{f}^{R}=0$ вытекает непосредственно из следствия 3. Пусть теперь $e_{f}^{R}=0$, покажем, что $b_{f}^{R}=1$. Если предположить, что $b_{f}^{R} \geqslant 2$, то, по определению правого барьера длины $b_{f}^{R}$, существуют такие наборы $\mathbf{x} \in V_{n-1}$, $\mathbf{x}^{\prime}, \mathbf{x}^{\prime \prime} \in V_{b_{f}^{R}-1}, \mathbf{x}^{\prime} \neq \mathbf{x}^{\prime \prime}, \mathbf{y} \in V_{b_{f}^{R}-1}$, что

$$
f_{b_{f}^{R}-1, n-1}^{\mathbf{x}}\left(\mathbf{x}^{\prime}\right)=f_{b_{f}^{R}-1, n-1}^{\mathbf{x}}\left(\mathbf{x}^{\prime \prime}\right)=\mathbf{y}
$$

Поэтому

$$
\left|f_{b_{f}^{R}-1, n-1}^{\mathbf{x}}-1(\mathbf{y})\right| \geqslant 2>2^{0}=2^{e_{f}^{R}},
$$

что противоречит теореме 4 .

Предложение 2. В условии теоремы 4 нельзя отказаться от условия

$$
l \geqslant b_{f}^{R}+p-n .
$$

Доказательство. Для доказательства достаточно рассмотреть функцию

$$
f\left(x_{1}, x_{2}, x_{3}, x_{4}, x_{5}\right)=x_{1} x_{2} x_{4} x_{5} \oplus x_{2} x_{4} x_{5} \oplus x_{1} x_{2} x_{3} x_{5} \oplus x_{1} x_{2} x_{5} \oplus x_{2} x_{5} \oplus x_{3} x_{5} \oplus x_{4}
$$

с правым барьером длины $b_{f}^{R}=4$ и заметить, что при $p=4=n-1, l=2<3=b_{f}^{R}-1$ выполнено соотношение

$$
\left|f_{2,4}^{(0,0,1,0)^{-1}}(0,0)\right|=1 \neq 2=\left|f_{2,4}^{(0,0,1,0)^{-1}}(1,1)\right| .
$$

При $p \geqslant 5$ ситуация аналогична.

Предложение 3. В условии теоремь 4 нельзя отказаться от условия $p \geqslant n-1$. 
Доказательство. Для функции $f$ с правым барьером длины $b_{f}^{R}=4$, определяемой согласно (17), при $p=3<4=n-1$ и $l=3=b_{f}^{R}-1$ получаем, что

$$
\left|f_{3,3}^{(0,1,0)^{-1}}(0,0,0)\right|=2 \neq 4=\left|f_{3,3}^{(0,1,0)^{-1}}(1,1,1)\right| \text {. }
$$

Необходимо также отметить, что в предложениях 2 и 3 для рассмотренных случаев в контрпримерах опровергнуты и более слабые утверждения, допускающие (в обозначениях теоремы 4) зависимость $e_{f}^{R}$ от $l, p$ и набора $\left(\tilde{x}_{1}, \tilde{x}_{2}, \ldots, \tilde{x}_{p}\right) \in V_{p}$.

Лемма 3. Пусть $f \in \mathscr{F}_{n}$ имеет правый барьер. Тогда для любого $l \geqslant b_{f}^{R}-1$ и любых наборов $\left(\tilde{x}_{1}, \tilde{x}_{2}, \ldots, \tilde{x}_{n-1}\right) \in V_{n-1},\left(y_{1}, y_{2}, \ldots, y_{l}\right) \in V_{l}$, если непусто множество $f_{l, n-1}^{\left(\tilde{x}_{1}, \tilde{x}_{2}, \ldots, \tilde{x}_{n-1}\right)^{-1}}\left(y_{1}, y_{2}, \ldots, y_{l}\right)$, то и для любых $l^{\prime} \geqslant l u\left(y_{l+1}, y_{l+2}, \ldots, y_{l^{\prime}}\right) \in V_{l^{\prime}-l}$ множество $f_{l^{\prime}, n-1}^{\left(\tilde{x}_{1}, \tilde{x}_{2}, \ldots, \tilde{x}_{n-1}\right)^{-1}}\left(y_{1}, y_{2}, \ldots, y_{l^{\prime}}\right)$ также непусто.

Доказательство. Проведем доказательство индукцией по $l^{\prime}, l^{\prime}=l, l+1, l+2, \ldots$ При $l^{\prime}=l$ утверждение верно. Предположим, что утверждение верно для $l^{\prime}=l^{*}$, докажем его для $l^{\prime}=l^{*}+1$. Из предположения и из теоремы 4 следует, что

$$
\left|f_{l^{*}, n-1}^{\left(\tilde{x}_{1}, \tilde{x}_{2}, \ldots, \tilde{x}_{n-1}\right)^{-1}}\left(y_{1}, y_{2}, \ldots, y_{l^{*}}\right)\right|=2^{e_{f}^{R}} .
$$

В случае, если

$$
\left|f_{l^{*}+1, n-1}^{\left(\tilde{x}_{1}, \tilde{x}_{2}, \ldots, \tilde{x}_{n-1}\right)^{-1}}\left(y_{1}, y_{2}, \ldots, y_{l^{*}+1}\right)\right|=0,
$$

то для каждого из $2^{e_{f}^{R}}$ наборов $\left(x_{1}, x_{2}, \ldots, x_{l^{*}}\right) \in f_{l^{*}, n-1}^{\left(\tilde{x}_{1}, \tilde{x}_{2}, \ldots, \tilde{x}_{n-1}\right)^{-1}}\left(y_{1}, y_{2}, \ldots, y_{l^{*}}\right)$ справедливо равенство

$$
\begin{aligned}
f_{l^{*}+1, n-1}^{\left(\tilde{x}_{1}, \tilde{x}_{2}, \ldots, \tilde{x}_{n-1}\right)}\left(x_{1}, x_{2}, \ldots, x_{l^{*}}, 0\right) & =f_{l^{*}+1, n-1}^{\left(\tilde{x}_{1}, \tilde{x}_{2}, \ldots, \tilde{x}_{n-1}\right)}\left(x_{1}, x_{2}, \ldots, x_{l^{*}}, 1\right) \\
& =\left(y_{1}, y_{2}, \ldots, y_{l^{*}}, y_{l^{*}+1} \oplus 1\right),
\end{aligned}
$$

поэтому

$$
\left|f_{l^{*}+1, n-1}^{\left(\tilde{x}_{1}, \tilde{x}_{2}, \ldots, \tilde{x}_{n-1}\right)^{-1}}\left(y_{1}, y_{2}, \ldots, y_{l^{*}+1} \oplus 1\right)\right|=2 \cdot 2^{e_{f}^{R}}
$$

что противоречит доказанному в теореме 4 .

Лемма 4. Пусть $f \in \mathscr{F}_{n}, m \geqslant 1$. Если наборь

$$
\begin{aligned}
\left(\tilde{x}_{1}, \tilde{x}_{2}, \ldots, \tilde{x}_{n-1}\right) & \in V_{n-1}, & & \left(y_{1}, y_{2}, \ldots, y_{m}\right) \\
\left(x_{1}^{\prime}, x_{2}^{\prime}, \ldots, x_{m}^{\prime}\right) & \in V_{m}, & \left(x_{1}^{\prime \prime}, x_{2}^{\prime \prime}, \ldots, x_{m}^{\prime \prime}\right) & \in V_{m}
\end{aligned}
$$

таковы, что $x_{1}^{\prime} \neq x_{1}^{\prime \prime} u$

$$
\begin{aligned}
\left(y_{1}, y_{2}, \ldots, y_{m}\right) & =f_{m, n-1}^{\left(\tilde{x}_{1}, \tilde{x}_{2}, \ldots, \tilde{x}_{n-1}\right)}\left(x_{1}^{\prime}, x_{2}^{\prime}, \ldots, x_{m}^{\prime}\right) \\
& =f_{m, n-1}^{\left(\tilde{x}_{1}, \tilde{x}_{2}, \ldots, \tilde{x}_{n-1}\right)}\left(x_{1}^{\prime \prime}, x_{2}^{\prime \prime}, \ldots, x_{m}^{\prime \prime}\right),
\end{aligned}
$$

mo

$$
\left|S_{1}\right|+\left|S_{2}\right|=|S|
$$


где

$$
\begin{aligned}
S & =f_{m, n-1}^{\left(\tilde{x}_{1}, \tilde{x}_{2}, \ldots, \tilde{x}_{n-1}\right)^{-1}}\left(y_{1}, y_{2}, \ldots, y_{m}\right), \\
S_{1} & =f_{m-1, n-1}^{\left(\tilde{x}_{2}, \tilde{x}_{3}, \ldots, \tilde{x}_{n-1}, x_{1}^{\prime}\right)^{-1}}\left(y_{2}, y_{3}, \ldots, y_{m}\right), \\
S_{2} & =f_{m-1, n-1}^{\left(\tilde{x}_{2}, \tilde{x}_{3}, \ldots, \tilde{x}_{n-1}, x_{1}^{\prime \prime}\right)^{-1}}\left(y_{2}, y_{3}, \ldots, y_{m}\right) .
\end{aligned}
$$

При этом $\left|S_{1}\right| \neq 0,\left|S_{2}\right| \neq 0$.

Доказательство. Рассмотрим множества

$$
\begin{aligned}
& S_{1}^{\prime}=\left\{\left(t_{1}, t_{2}, t_{3}, \ldots, t_{m}\right) \mid t_{1}=x_{1}^{\prime},\left(t_{2}, t_{3}, \ldots, t_{m}\right) \in S_{1}\right\}, \\
& S_{2}^{\prime}=\left\{\left(t_{1}, t_{2}, t_{3}, \ldots, t_{m}\right) \mid t_{1}=x_{1}^{\prime \prime},\left(t_{2}, t_{3}, \ldots, t_{m}\right) \in S_{2}\right\} .
\end{aligned}
$$

Очевидно, что

$$
\left|S_{1}^{\prime}\right|=\left|S_{1}\right|, \quad\left|S_{2}^{\prime}\right|=\left|S_{2}\right| .
$$

Множества $S_{1}^{\prime}$ и $S_{2}^{\prime}$ непусты, так как $\left(x_{1}^{\prime}, x_{2}^{\prime}, \ldots, x_{m}^{\prime}\right) \in S_{1}^{\prime},\left(x_{1}^{\prime \prime}, x_{2}^{\prime \prime}, \ldots, x_{m}^{\prime \prime}\right) \in S_{2}^{\prime}$; при этом $S_{1}^{\prime} \cap S_{2}^{\prime}=\varnothing$, так как $x_{1}^{\prime} \neq x_{1}^{\prime \prime}$. Кроме того, $S_{1}^{\prime} \cup S_{2}^{\prime}=S$, так как $\left\{x_{1}^{\prime}, x_{1}^{\prime \prime}\right\}=\{0,1\}$. Отсюда получаем равенство $\left|S_{1}^{\prime}\right|+\left|S_{2}^{\prime}\right|=|S|$.

Теорема 5. Функция $f \in \mathscr{F}_{n}$ имеет правый барьер тогда и только тогда, когда существуют натуральные числа $b$ и с такие, что для любого $l \geqslant b-1$ и любых наборов $\mathbf{y} \in V_{l}, \mathbf{x} \in A_{\mathbf{y}}^{f}$ справедливо равенство

$$
\left|f_{l, n-1}^{\mathbf{x}}-1(\mathbf{y})\right|=c .
$$

Доказательство. Необходимость $\left(b=b_{f}^{R}, c=2^{e_{f}^{R}}\right)$ доказана в теореме 4. Чтобы доказать достаточность, покажем, что для произвольной $f \in \mathscr{F}_{n}$ без правого барьера и любого сколь угодно большого $b$ существуют $l^{\prime}, l^{\prime \prime} \geqslant b-1, l^{\prime \prime}=l^{\prime}-1$, и наборы $\left(\tilde{x}_{1}, \tilde{x}_{2}, \ldots, \tilde{x}_{n}\right) \in$ $V_{n}$ и $\left(y_{1}, y_{2}, \ldots, y_{l^{\prime}}\right) \in V_{l^{\prime}}$, такие, что

$$
\begin{aligned}
S_{1} & =f_{l^{\prime \prime}, n-1}^{\left(\tilde{x}_{2}, \tilde{x}_{3}, \ldots, \tilde{x}_{n-1}, \tilde{x}_{n}\right)^{-1}}\left(y_{2}, y_{3}, \ldots, y_{l^{\prime}}\right) \neq \varnothing, \\
S & =f_{l^{\prime}, n-1}^{\left(\tilde{x}_{1}, \tilde{x}_{2}, \ldots, \tilde{x}_{n-1}\right)^{-1}}\left(y_{1}, y_{2}, \ldots, y_{l^{\prime}}\right) \neq \varnothing, \quad|S| \neq\left|S_{1}\right| .
\end{aligned}
$$

Положим $l^{\prime \prime}=b-1, l^{\prime}=b$. По определению функции без правого барьера, существуют наборы $\left(x_{1}, x_{2}, \ldots, x_{n-1}\right) \in V_{n-1},\left(x_{1}^{\prime}, x_{2}^{\prime}, \ldots, x_{l^{\prime}}^{\prime}\right),\left(x_{1}^{\prime \prime}, x_{2}^{\prime \prime}, \ldots, x_{l^{\prime}}^{\prime \prime}\right) \in V_{l^{\prime}}$ такие, что $x_{1}^{\prime} \neq x_{1}^{\prime \prime}$ и

$$
f_{l^{\prime}, n-1}^{\left(x_{1}, x_{2}, \ldots, x_{n-1}\right)}\left(x_{1}^{\prime}, x_{2}^{\prime}, \ldots, x_{l^{\prime}}^{\prime}\right)=f_{l^{\prime}, n-1}^{\left(x_{1}, x_{2}, \ldots, x_{n-1}\right)}\left(x_{1}^{\prime \prime}, x_{2}^{\prime \prime}, \ldots, x_{l^{\prime}}^{\prime \prime}\right) .
$$

Положим

$$
\begin{aligned}
\left(y_{1}, y_{2}, \ldots, y_{l^{\prime}}\right) & =f_{l^{\prime}, n-1}^{\left(x_{1}, x_{2}, \ldots, x_{n-1}\right)}\left(x_{1}^{\prime}, x_{2}^{\prime}, \ldots, x_{l^{\prime}}^{\prime}\right), \\
\left(\tilde{x}_{1}, \tilde{x}_{2}, \ldots, \tilde{x}_{n}\right) & =\left(x_{1}, x_{2}, \ldots, x_{n-1}, x_{1}^{\prime}\right) .
\end{aligned}
$$

Тогда по лемме 4

$$
\left|S_{1}\right|+\left|S_{2}\right|=|S|, \quad\left|S_{1}\right|>0, \quad\left|S_{2}\right|>0,
$$

отсюда $|S| \neq\left|S_{1}\right|$, и (18) доказано. 
Лемма 5. Если $f \in \mathscr{F}_{n}$ имеет правый барьер и $e_{f}^{R}<b_{f}^{R}-1$, то существуют наборы $\mathbf{x}^{*}, \mathbf{x}^{* *} \in V_{n-1}, \mathbf{y}^{*}, \mathbf{y}^{* *} \in V_{b_{f}^{R}-2}$ такие, что

$$
\left|f_{b_{f}^{R}-2, n-1}^{\mathbf{x}^{*}}-1\left(\mathbf{y}^{*}\right)\right| \neq\left|f_{b_{f}^{R}-2, n-1}^{\mathbf{x}^{* *}}{ }^{-1}\left(\mathbf{y}^{* *}\right)\right| .
$$

Доказательство. По определению правого барьера длины $b_{f}^{R}$, существуют такие наборы

$$
\begin{array}{ll}
\mathbf{x}^{\prime}=\left(x_{1}^{\prime}, x_{2}^{\prime}, \ldots, x_{n-1}^{\prime}\right) \in V_{n-1}, & \mathbf{t}^{\prime}=\left(t_{1}^{\prime}, t_{2}^{\prime}, \ldots, t_{b_{f}^{R}-1}^{\prime}\right) \in V_{b_{f}^{R}-1}, \\
\mathbf{t}^{\prime \prime}=\left(t_{1}^{\prime \prime}, t_{2}^{\prime \prime}, \ldots, t_{b_{f}^{R}-1}^{\prime \prime}\right) \in V_{b_{f}^{R}-1}, & \mathbf{y}^{\prime}=\left(y_{1}^{\prime}, y_{2}^{\prime}, \ldots, y_{b_{f}^{R}-1}^{\prime}\right) \in V_{b_{f}^{R}-1},
\end{array}
$$

что $t_{1}^{\prime} \neq t_{1}^{\prime \prime}$ и

$$
f_{b_{f}^{R}-1, n-1}^{\mathbf{x}^{\prime}}\left(\mathbf{t}^{\prime}\right)=f_{b_{f}^{R}-1, n-1}^{\mathbf{x}^{\prime}}\left(\mathbf{t}^{\prime \prime}\right)=\mathbf{y}^{\prime}
$$

По лемме 4,

$$
\begin{aligned}
\mid f_{b_{f}^{R}-1, n-1}^{\mathbf{x}^{\prime}}-1 & \left(\mathbf{y}^{\prime}\right)|=| f_{b_{f}^{R}-2, n-1}^{\left(x_{2}^{\prime}, x_{3}^{\prime}, \ldots, x_{n-1}^{\prime}, t_{1}^{\prime}\right)^{-1}}\left(y_{2}^{\prime}, y_{3}^{\prime}, \ldots, y_{b_{f}^{R}-1}^{\prime}\right) \mid \\
& +\left|f_{b_{f}^{R}-2, n-1}^{\left(x_{2}^{\prime}, x_{3}^{\prime}, \ldots, x_{n-1}^{\prime}, t_{1}^{\prime \prime}\right)^{-1}}\left(y_{2}^{\prime}, y_{3}^{\prime}, \ldots, y_{b_{f}^{R}-1}^{\prime}\right)\right|
\end{aligned}
$$

причем мощности всех множеств в последнем соотношении не равны нулю. Положим

$$
\begin{aligned}
& \mathbf{x}^{*}=\left(x_{2}^{\prime}, x_{3}^{\prime}, \ldots, x_{n-1}^{\prime}, t_{1}^{\prime}\right), \\
& \mathbf{y}^{*}=\left(y_{2}^{\prime}, y_{3}^{\prime}, \ldots, y_{b_{f}^{R}-1}^{\prime}\right) .
\end{aligned}
$$

Тогда

$$
\left|f_{b_{f}^{R}-2, n-1}^{\mathbf{x}^{*}}-1\left(\mathbf{y}^{*}\right)\right|<\left|f_{b_{f}^{R}-1, n-1}^{\mathbf{x}^{\prime}}-1\left(\mathbf{y}^{\prime}\right)\right|
$$

Так как $e_{f}^{R}<b_{f}^{R}-1$, то, по следствию $3, f$ зависит хотя бы от одной из последних $b_{f}^{R}-1$ переменных. Поэтому, как легко показать, существуют наборы

$$
\begin{aligned}
& \mathbf{x}^{\prime \prime}=\left(x_{1}^{\prime \prime}, x_{2}^{\prime \prime}, \ldots, x_{n-1}^{\prime \prime}\right) \in V_{n-1}, \\
& \mathbf{y}^{\prime \prime}=\left(y_{1}^{\prime \prime}, y_{2}^{\prime \prime}, \ldots, y_{b_{f}^{R}-1}^{\prime \prime}\right) \in V_{b_{f}^{R}-1},
\end{aligned}
$$

а также $t \in\{0,1\}$ такие, что множество $f_{b_{f}^{R}-1, n-1}^{\mathbf{x}^{\prime \prime}}\left(\mathbf{y}^{\prime \prime}\right)$ непусто, причем для любого набора $\left(t_{1}, t_{2}, \ldots, t_{b_{f}^{R}-1}\right) \in f_{b_{f}^{R}-1, n-1}^{\mathbf{x}^{\prime \prime}}\left(\mathbf{y}^{\prime \prime}\right)$ справедливо равенство $t_{1}=t$. Пусть

$$
\begin{aligned}
& \mathbf{x}^{* *}=\left(x_{2}^{\prime \prime}, x_{3}^{\prime \prime}, \ldots, x_{n-1}^{\prime \prime}, t\right), \\
& \mathbf{y}^{* *}=\left(y_{2}^{\prime \prime}, y_{3}^{\prime \prime}, \ldots, y_{b_{f}^{R}-1}^{\prime \prime}\right) .
\end{aligned}
$$

Тогда

$$
\left|f_{b_{f}^{R}-2, n-1}^{\mathbf{x}^{* *}}-1\left(\mathbf{y}^{* *}\right)\right|=\left|f_{b_{f}^{R}-1, n-1}^{\mathbf{x}^{\prime \prime}}-1\left(\mathbf{y}^{\prime \prime}\right)\right|
$$


Как следует из теоремы 4,

$$
\left|f_{b_{f}^{R}-1, n-1}^{\mathbf{x}^{\prime}}{ }^{-1}\left(\mathbf{y}^{\prime}\right)\right|=\left|f_{b_{f}^{R}-1, n-1}^{\mathbf{x}^{\prime \prime}}{ }^{-1}\left(\mathbf{y}^{\prime \prime}\right)\right|=2^{e_{f}^{R}},
$$

из чего, с учетом (19) и (20), следует, что

$$
\left.\left|f_{b_{f}^{R}-2, n-1}^{\mathbf{x}^{*}}\left(\mathbf{y}^{*}\right)\right| \neq \mid f_{b_{f}^{R}-2, n-1}^{\mathbf{x}^{* *}}-1 \mathbf{y}^{* *}\right) \mid .
$$

Из леммы 5 и следствия 3 легко получить следующее утверждение.

Следствие 4. В условии теоремь 4 ни для одной функции нельзя ослабить ограничение $l \geqslant b_{f}^{R}+p-n$.

Теорема 6. Пусть $f \in \mathscr{F}_{n}$ не имеет правого барьера. Тогда для любого сколь угодно большого $l$ существует такое $k$ и такие наборы $\mathbf{x}^{*} \in V_{n-1},\left(y_{1}^{*}, y_{2}^{*}, \ldots, y_{l}^{*}\right) \in \operatorname{Im}\left(f_{l, n-1}^{\mathbf{x}^{*}}\right)$, $\left(a_{1}^{\prime}, a_{2}^{\prime}, \ldots, a_{k}^{\prime}\right),\left(a_{1}^{\prime \prime}, a_{2}^{\prime \prime}, \ldots, a_{k}^{\prime \prime}\right) \in V_{k}$, что

$$
\left|f_{l+k, n-1}^{\mathbf{x}^{*}-1}\left(y_{1}^{*}, y_{2}^{*}, \ldots, y_{l}^{*}, a_{1}^{\prime}, a_{2}^{\prime}, \ldots, a_{k}^{\prime}\right)\right| \neq\left|f_{l+k, n-1}^{\mathbf{x}^{*}-1}\left(y_{1}^{*}, y_{2}^{*}, \ldots, y_{l}^{*}, a_{1}^{\prime \prime}, a_{2}^{\prime \prime}, \ldots, a_{k}^{\prime \prime}\right)\right| .
$$

Доказательство. Ясно, что достаточно рассмотреть случай $l \geqslant n-1$. Функция $f$ не имеет правого барьера, поэтому для любого $l$ существуют наборы $\left(x_{0}, x_{1}, \ldots, x_{n-2}\right) \in V_{n-1}$, $\left(t_{0}^{\prime}, t_{1}^{\prime}, \ldots, t_{l}^{\prime}\right),\left(t_{0}^{\prime \prime}, t_{1}^{\prime \prime}, \ldots, t_{l}^{\prime \prime}\right),\left(y_{0}^{*}, y_{1}^{*}, \ldots, y_{l}^{*}\right) \in V_{l+1}$ такие, что

$$
\begin{aligned}
f_{l+1, n-1}^{\left(x_{0}, x_{1}, \ldots, x_{n-2}\right)}\left(t_{0}^{\prime}, t_{1}^{\prime}, \ldots, t_{l}^{\prime}\right) & \\
= & \left(y_{0}^{*}, y_{1}^{*}, \ldots, y_{l}^{*}\right)=f_{l+1, n-1}^{\left(x_{0}, x_{1}, \ldots, x_{n-2}\right)}\left(t_{0}^{\prime \prime}, t_{1}^{\prime \prime}, \ldots, t_{l}^{\prime \prime}\right), \quad t_{0}^{\prime} \neq t_{0}^{\prime \prime} .
\end{aligned}
$$

Положим $\mathbf{x}^{*}=\left(x_{1}, \ldots, x_{n-2}, t_{0}^{\prime}\right)$. Из леммы 4 следует неравенство

$$
\left|f_{l, n-1}^{\mathbf{x}^{*}}-1\left(y_{1}^{*}, \ldots, y_{l}^{*}\right)\right|<\left|f_{l+1, n-1}^{\left(x_{0}, x_{1}, \ldots, x_{n-2}\right)^{-1}}\left(y_{0}^{*}, y_{1}^{*}, \ldots, y_{l}^{*}\right)\right| .
$$

Предположим противное: для любых $k$ и $\left(a_{1}^{\prime}, a_{2}^{\prime}, \ldots, a_{k}^{\prime}\right),\left(a_{1}^{\prime \prime}, a_{2}^{\prime \prime}, \ldots, a_{k}^{\prime \prime}\right) \in V_{k}$ справедливо равенство

$$
\begin{aligned}
& \left|f_{l+k, n-1}^{\mathbf{x}^{*}}{ }^{-1}\left(y_{1}^{*}, y_{2}^{*}, \ldots, y_{l}^{*}, a_{1}^{\prime}, a_{2}^{\prime}, \ldots, a_{k}^{\prime}\right)\right| \\
& =\left|f_{l+k, n-1}^{\mathbf{x}^{*}}{ }^{-1}\left(y_{1}^{*}, y_{2}^{*}, \ldots, y_{l}^{*}, a_{1}^{\prime \prime}, a_{2}^{\prime \prime}, \ldots, a_{k}^{\prime \prime}\right)\right| .
\end{aligned}
$$

С помощью леммы 1 легко доказать по индукции, что из предположения (22) следует, что равенство

$$
\left|f_{l+k, n-1}^{\mathbf{x}^{*}}-1\left(y_{1}^{*}, y_{2}^{*}, \ldots, y_{l}^{*}, a_{1}, a_{2}, \ldots, a_{k}\right)\right|=\left|f_{l, n-1}^{\mathbf{x}^{*}-1}\left(y_{1}^{*}, y_{2}^{*}, \ldots, y_{l}^{*}\right)\right|=c
$$

верно для любого $k \geqslant 0$ и для любого набора $\left(a_{1}, a_{2}, \ldots, a_{k}\right) \in V_{k}$.

Выберем из множества $f_{l, n-1}^{\mathbf{x}^{*}}-1\left(y_{1}^{*}, y_{2}^{*}, \ldots, y_{l}^{*}\right)$ произвольный набор $\left(t_{1}^{*}, t_{2}^{*}, \ldots, t_{l}^{*}\right)$ и рассмотрим $k=n+l$ и набор $\left(a_{1}^{*}, a_{2}^{*}, \ldots, a_{n+l}^{*}\right) \in V_{n+l}$ такой, что

$$
\begin{aligned}
\left(a_{1}^{*}, a_{2}^{*}, \ldots, a_{n-1}^{*}\right) & =f_{n-1}\left(t_{l-n+2}^{*}, t_{l-n+3}^{*}, \ldots, t_{l}^{*}, x_{0}, x_{1}, \ldots, x_{n-2}\right), \\
\left(a_{n}^{*}, a_{n+1}^{*}, \ldots, a_{n+l}^{*}\right) & =\left(y_{0}^{*}, y_{1}^{*}, \ldots, y_{l}^{*}\right) .
\end{aligned}
$$


Справедливо включение

$$
\begin{array}{r}
\left\{\left(z_{1}, z_{2}, \ldots, z_{l}, b_{0}, b_{1}, \ldots, b_{n-2}, t_{0}, t_{1}, \ldots, t_{l}\right) \in V_{l+n+l} \mid\left(z_{1}, z_{2}, \ldots, z_{l}\right)=\left(t_{1}^{*}, t_{2}^{*}, \ldots, t_{l}^{*}\right),\right. \\
\left.\left(b_{0}, b_{1}, \ldots, b_{n-2}\right)=\left(x_{0}, x_{1}, \ldots, x_{n-2}\right),\left(t_{0}, t_{1}, \ldots, t_{l}\right) \in f_{l+1, n-1}^{\left(x_{0}, x_{1}, \ldots, x_{n-2}\right)^{-1}}\left(y_{0}^{*}, y_{1}^{*}, \ldots, y_{l}^{*}\right)\right\} \\
\subseteq f_{l+n+l, n-1}^{\mathbf{x}^{*}}\left(y_{1}^{*}, y_{2}^{*}, \ldots, y_{l}^{*}, a_{1}^{*}, a_{2}^{*}, \ldots, a_{n+l}^{*}\right),
\end{array}
$$

из которого легко получить неравенство

$$
\left|f_{l+n+l, n-1}^{\mathbf{x}^{*}}\left(y_{1}^{*}, y_{2}^{*}, \ldots, y_{l}^{*}, a_{1}^{*}, a_{2}^{*}, \ldots, a_{n+l}^{*}\right)\right| \geqslant\left|f_{l+1, n-1}^{\left(x_{0}, x_{1}, \ldots, x_{n-2}\right)^{-1}}\left(y_{0}^{*}, y_{1}^{*}, \ldots, y_{l}^{*}\right)\right| .
$$

Учитывая (21), (23) и (24), получим следующую цепочку соотношений:

$$
\begin{aligned}
c & =\mid f_{l+n+l, n-1}^{\mathbf{x}^{*}}-1 \\
& \geqslant\left|f_{l+1, n-1}^{\left(x_{0}, x_{1}, \ldots, x_{n-2}\right)^{-1}}\left(y_{0}^{*}, y_{1}^{*}, \ldots, y_{l}^{*}, a_{1}^{*}, a_{2}^{*}, \ldots, a_{l}^{*}\right)\right| \\
& >\left|f_{l, n-1}^{\mathbf{x}^{*}-1}\left(y_{1}^{*}, \ldots, y_{l}^{*}\right)\right|=c .
\end{aligned}
$$

Полученное противоречие завершает доказательство теоремы.

Для любой последовательности случайных величин $\left\{\xi_{i}\right\}_{i=1}^{\infty}$ введем следующее обозначение: для любого натурального $i$ через $\Xi_{i}$ будем обозначать случайный вектор $\left(\xi_{1}, \xi_{2}, \ldots, \xi_{i}\right)$.

Определение 5. Пусть $\left\{\xi_{i}\right\}_{i=1}^{\infty}-$ чисто случайная последовательность случайных величин. Будем называть функцию $f \in \mathscr{F}_{n}$ функцией без предсказывания, если существует натуральное $b$ такое, что для любого $l \geqslant b-1$, любых наборов $\mathbf{x}^{*} \in V_{n-1}$, $\left(y_{1}^{*}, y_{2}^{*}, \ldots, y_{l}^{*}\right) \in \operatorname{Im}\left(f_{l, n-1}^{\mathbf{x}^{*}}\right)$ и любого $k$ выполняется равенство

$$
H\left(f_{l+k, n-1}^{\mathbf{x}^{*}}\left(\Xi_{l+k}\right) \mid f_{l, n-1}^{\mathbf{x}^{*}}\left(\Xi_{l}\right)=\left(y_{1}^{*}, y_{2}^{*}, \ldots, y_{l}^{*}\right)\right)=k .
$$

Кодирующее устройство с функцией усложнения, являющейся функцией без предсказывания, обладает следующим свойством. Пусть преобразовывается двоичная последовательность, известная вплоть до определенного символа и предполагаемая чисто случайной после него. В таком случае через некоторое число тактов работы кодирующего устройства наступает момент, после которого становится невозможным получить никакую информацию ни об одной выходной $k$-грамме (при любом $k$ ) по предшествующим этой $k$-грамме символам выходной последовательности.

Замечание 4. Используя элементарные свойства условной энтропии и тождество

$$
\begin{aligned}
& \mathbf{P}\left\{f_{l+k, n-1}^{\mathbf{x}^{*}}\left(\Xi_{l+k}\right)=\left(y_{1}^{*}, \ldots, y_{l}^{*}, a_{1}, \ldots, a_{k}\right) \mid f_{l, n-1}^{\mathbf{x}^{*}}\left(\Xi_{l}\right)=\left(y_{1}^{*}, \ldots, y_{l}^{*}\right)\right\} \\
& \equiv \mathbf{P}\left\{f_{l+k, n-1}^{\mathbf{x}^{*}}\left(\Xi_{l+k}\right)=\left(y_{1}^{*}, \ldots, y_{l}^{*}, a_{1}, \ldots, a_{k}\right) \mid f_{l+k-1, n-1}^{\mathbf{x}^{*}}\left(\Xi_{l+k-1}\right)\right. \\
& \left.\quad=\left(y_{1}^{*}, \ldots, y_{l}^{*}, a_{1}, \ldots, a_{k-1}\right)\right\} \\
& \quad \times \mathbf{P}\left\{f_{l+k-1, n-1}^{\mathbf{x}^{*}}\left(\Xi_{l+k-1}\right)=\left(y_{1}^{*}, \ldots, y_{l}^{*}, a_{1}, \ldots, a_{k-1}\right) \mid f_{l+k-2, n-1}^{\mathbf{x}^{*}}\left(\Xi_{l+k-2}\right)\right. \\
& \left.\quad=\left(y_{1}^{*}, \ldots, y_{l}^{*}, a_{1}, \ldots, a_{k-2}\right)\right\} \\
& \quad \times \ldots \times \mathbf{P}\left\{f_{l+1, n-1}^{\mathbf{x}^{*}}\left(\Xi_{l+1}\right)=\left(y_{1}^{*}, \ldots, y_{l}^{*}, a_{1}\right) \mid f_{l, n-1}^{\mathbf{x}^{*}}\left(\Xi_{l}\right)=\left(y_{1}^{*}, \ldots, y_{l}^{*}\right)\right\},
\end{aligned}
$$


верное для любого $k$, нетрудно показать, что множество функций без предсказывания не изменится, если в определении 5 требовать выполнения условия (25) только при $k=1$, а не при произвольном $k$. Таким образом, функция удовлетворяет определению 5 тогда и только тогда, когда после определенного момента времени любой очередной символ выходной последовательности кодирующего устройства с данной функцией усложнения (преобразовывающего чисто случайную последовательность) становится невозможно предсказать (с вероятностью, отличной от 1/2) по предшествующим ему символам выходной последовательности и началу входной последовательности.

Заметим, что совершенная уравновешенность есть необходимое условие принадлежности функции классу функций без предсказывания. Это легко показать с использованием теоремы 1 , положив для любой $f \notin \mathscr{P} \mathscr{B}_{n}$ (то есть, функции с запретом) в соотношении (25) для сколь угодно большого $l$ в качестве $\left(y_{1}^{*}, y_{2}^{*}, \ldots, y_{l}^{*}\right)$ любой набор такой, что $f_{l}^{-1}\left(y_{1}^{*}, y_{2}^{*}, \ldots, y_{l}^{*}\right) \neq \varnothing$ и $f_{l+1}^{-1}\left(y_{1}^{*}, y_{2}^{*}, \ldots, y_{l}^{*}, t\right)=\varnothing$ для некоторого $t \in\{0,1\}$. Существование такого набора при сколь угодно большом $l$ для любой функции с запретом доказывается тривиально, и равенство (25) (например, при $k=1$ ) для такого набора, очевидно, не выполняется.

Чтобы показать, что данное необходимое условие не является достаточным, приведем пример совершенно уравновешенной булевой функции, не являющейся функцией без предсказывания.

Пример 1. Пусть

$$
f\left(x_{1}, x_{2}, x_{3}\right)=x_{1} \oplus x_{2} x_{3}
$$

Тогда при сколь угодно большом $l$

$$
\begin{aligned}
H\left(f_{l+2,2}^{(1,1)}\left(\Xi_{l+2}\right) \mid f_{l, 2}^{(1,1)}\left(\Xi_{l}\right)=(0,0, \ldots, 0)\right) & =H\left(f_{2,2}^{(1,1)}\left(\xi_{l+1}, \xi_{l+2}\right)\right) \\
& =-\left(\frac{1}{2} \log _{2} \frac{1}{2}+\frac{1}{4} \log _{2} \frac{1}{4}+\frac{1}{4} \log _{2} \frac{1}{4}\right) \\
& =\frac{3}{2}<2 .
\end{aligned}
$$

Как показано в [5], не являющаяся функцией без предсказывания функция $f$ в последнем примере не имеет правого барьера; легко видеть также, что любая функция с правым барьером длины 1 , то есть, линейная по последней переменной, является функцией без предсказывания. Сформулируем и докажем утверждение, полностью описывающее класс функций без предсказывания в терминах барьеров.

Теорема 7. Функция $f \in \mathscr{F}_{n}$ является функичей без предсказывания тогда и только тогда, когда имеет правый барьер.

Доказательство. Пусть $\left\{\xi_{i}\right\}_{i=1}^{\infty}-$ чисто случайная последовательность. Как нетрудно видеть, из свойств энтропии следует, что условие (25) эквивалентно тому, что для любого набора $\left(a_{1}, a_{2}, \ldots, a_{k}\right) \in V_{k}$ выполняется равенство

$$
\mathbf{P}\left\{f_{l+k, n-1}^{\mathbf{x}^{*}}\left(\Xi_{l+k}\right)=\left(y_{1}^{*}, \ldots, y_{l}^{*}, a_{1}, \ldots, a_{k}\right) \mid f_{l, n-1}^{\mathbf{x}^{*}}\left(\Xi_{l}\right)=\left(y_{1}^{*}, \ldots, y_{l}^{*}\right)\right\}=2^{-k} .
$$

Пусть $f$ имеет правый барьер, тогда покажем, что $f$ удовлетворяет определению 5 с $b=b_{f}^{R}$. По лемме 3 , для любых $k \geqslant 0, l \geqslant b_{f}^{R}-1$ и любых наборов $\mathbf{x}^{*} \in V_{n-1}$, $\left(y_{1}^{*}, y_{2}^{*}, \ldots, y_{l}^{*}\right) \in \operatorname{Im}\left(f_{l, n-1}^{\mathbf{x}^{*}}\right),\left(a_{1}, a_{2}, \ldots, a_{k}\right) \in V_{k}$ множество $f_{l+k, n-1}^{\mathbf{x}^{*}}\left(y_{1}^{*}, y_{2}^{*}, \ldots, y_{l}^{*}\right.$, 
$\left.a_{1}, a_{2}, \ldots, a_{k}\right)$ непусто, а значит, по теореме 4 , его мощность, так же, как и мощность множества $f_{l, n-1}^{\mathbf{x}^{*}-1}\left(y_{1}^{*}, y_{2}^{*}, \ldots, y_{l}^{*}\right)$, равна $2^{e_{f}^{R}}$. С учетом этого, а также свойств распределения случайных векторов $\Xi_{i}$, получим следующую цепочку равенств:

$$
\begin{aligned}
\mathbf{P}\left\{f_{l+k, n-1}^{\mathbf{x}^{*}}\left(\Xi_{l+k}\right)=\left(y_{1}^{*}, y_{2}^{*}, \ldots, y_{l}^{*}, a_{1}, a_{2}, \ldots, a_{k}\right) \mid f_{l, n-1}^{\mathbf{x}^{*}}\left(\Xi_{l}\right)=\left(y_{1}^{*}, y_{2}^{*}, \ldots, y_{l}^{*}\right)\right\} \\
=\frac{\mathbf{P}\left\{f_{l+k, n-1}^{\mathbf{x}^{*}}\left(\Xi_{l+k}\right)=\left(y_{1}^{*}, y_{2}^{*}, \ldots, y_{l}^{*}, a_{1}, a_{2}, \ldots, a_{k}\right)\right\}}{\mathbf{P}\left\{f_{l, n-1}^{\mathbf{x}^{*}}\left(\Xi_{l}\right)=\left(y_{1}^{*}, y_{2}^{*}, \ldots, y_{l}^{*}\right)\right\}} \\
=\frac{2^{e_{f}^{R}} / 2^{l+k}}{2_{f}^{e_{f}^{R}} / 2^{l}}=2^{-k} .
\end{aligned}
$$

Из произвольности набора $\left(a_{1}, a_{2}, \ldots, a_{k}\right) \in V_{k}$ следует требуемое утверждение для функций с правым барьером. Достаточность доказана.

Для доказательства необходимости заметим, что, согласно теореме 6 , в случае функции без правого барьера для любого сколь угодно большого $b$ существуют $k, l \geqslant b-1$ и наборы $\mathbf{x}^{*} \in V_{n-1},\left(y_{1}^{*}, y_{2}^{*}, \ldots, y_{l}^{*}\right) \in \operatorname{Im}\left(f_{l, n-1}^{\mathbf{x}^{*}}\right),\left(a_{1}^{\prime}, a_{2}^{\prime}, \ldots, a_{k}^{\prime}\right),\left(a_{1}^{\prime \prime}, a_{2}^{\prime \prime}, \ldots, a_{k}^{\prime \prime}\right) \in V_{k}$, такие, что

$$
\left|f_{l+k, n-1}^{\mathbf{x}^{*}-1}\left(y_{1}^{*}, y_{2}^{*}, \ldots, y_{l}^{*}, a_{1}^{\prime}, a_{2}^{\prime}, \ldots, a_{k}^{\prime}\right)\right| \neq\left|f_{l+k, n-1}^{\mathbf{x}^{*}-1}\left(y_{1}^{*}, y_{2}^{*}, \ldots, y_{l}^{*}, a_{1}^{\prime \prime}, a_{2}^{\prime \prime}, \ldots, a_{k}^{\prime \prime}\right)\right| \text {. }
$$

Тогда

$$
\begin{aligned}
& \mathbf{P}\left\{f_{l+k, n-1}^{\mathbf{x}^{*}}\left(\Xi_{l+k}\right)=\left(y_{1}^{*}, y_{2}^{*}, \ldots, y_{l}^{*}, a_{1}^{\prime}, a_{2}^{\prime}, \ldots, a_{k}^{\prime}\right) \mid f_{l, n-1}^{\mathbf{x}^{*}}\left(\Xi_{l}\right)=\left(y_{1}^{*}, y_{2}^{*}, \ldots, y_{l}^{*}\right)\right\} \\
& =\frac{\left|f_{l+k, n-1}^{\mathbf{x}^{*}}\left(y_{1}^{*}, y_{2}^{*}, \ldots, y_{l}^{*}, a_{1}^{\prime}, a_{2}^{\prime}, \ldots, a_{k}^{\prime}\right)\right| / 2^{l+k}}{\mathbf{P}\left\{f_{l, n-1}^{\mathbf{x}^{*}}\left(\Xi_{l}\right)=\left(y_{1}^{*}, y_{2}^{*}, \ldots, y_{l}^{*}\right)\right\}} \\
& \neq \frac{\left|f_{l+k, n-1}^{\mathbf{x}^{*}}\left(y_{1}^{*}, y_{2}^{*}, \ldots, y_{l}^{*}, a_{1}^{\prime \prime}, a_{2}^{\prime \prime}, \ldots, a_{k}^{\prime \prime}\right)\right| / 2^{l+k}}{\mathbf{P}\left\{f_{l, n-1}^{\mathbf{x}^{*}}\left(\Xi_{l}\right)=\left(y_{1}^{*}, y_{2}^{*}, \ldots, y_{l}^{*}\right)\right\}} \\
& =\mathbf{P}\left\{f_{l+k, n-1}^{\mathbf{x}^{*}}\left(\Xi_{l+k}\right)=\left(y_{1}^{*}, y_{2}^{*}, \ldots, y_{l}^{*}, a_{1}^{\prime \prime}, a_{2}^{\prime \prime}, \ldots, a_{k}^{\prime \prime}\right) \mid f_{l, n-1}^{\mathbf{x}^{*}}\left(\Xi_{l}\right)=\left(y_{1}^{*}, y_{2}^{*}, \ldots, y_{l}^{*}\right)\right\},
\end{aligned}
$$

что завершает доказательство необходимости.

\section{Список литературы}

1. Golić D. J., On the security of nonlinear filter generators. Lecture Notes Computer Sci. (1996) 1039, 173-188.

2. Сумароков С. Н., Запреты двоичных функций и обратимость для одного класса кодирующих устройств. Обозрение прикладной и промышленной математики (1994) 1, №1, 33-55.

3. Логачев О. А., Об одном классе совершенно уравновешенных булевых функций. В сб.: Материаль 3-й Международной научной конференции по проблемам безопасности и противодействия терроризму. МЦНМО, Москва, 2008, с. 137-141.

4. Логачев О. А., Смышляев С. В., Ященко В. В., Новые методы изучения совершенно уравновешенных булевых функций. Дискретная математика (2009) 21, №2, 51-74.

5. Смышляев С. В., О некоторых свойствах совершенно уравновешенных булевых функций. В сб.: Материалы 4-й Международной научной конференции по проблемам безопасности и противодействия терроризму. МЦНМО, Москва, 2009, с. 57-64. 
6. Смышляев С. В., О совершенно уравновешенных булевых функциях без барьера. В сб.: Материаль 8-й Международной научной конференщии «Дискретные модели в теории управляюших систем». МАКС Пресс, Москва, 278-284.

7. Смышляев С. В., Барьеры совершенно уравновешенных булевых функций. Дискретная математика (2010) 22, №2, 66-79.

8. Смышляев С. В., О преобразовании двоичных последовательностей с помощью совершенно уравновешенных булевых функций. В сб.: Материаль 5-й Международной научной конференции по проблемам безопасности и противодействия терроризму. МЦНМО, Москва, 2010, c. $31-41$.

9. Смышляев С. В., О криптографических слабостях некоторых классов преобразований двоичных последовательностей. Прикладная дискретная математика (2010) 7, №1, 5-15.

Статья поступила 3.09.2010. 